\title{
Diferencias neuroanatómicas y funcionales entre razonamiento numérico y cálculo: evidencia de doble disociación
}

\author{
María Pilar Salguero Alcañiz y José Ramón Alameda Bailén \\ Universidad de Huelva \\ Correspondencia: José Ramón Alameda Bailén. \\ Área de Psicología Básica. Facultad de Ciencias de la Educación. Campus de El Carmen. \\ Avda. Fuerzas Armadas, 3 - 21071 Huelva. Teléfono: 959219 217. Correo electrónico: alameda@uhu.es
}

\section{RESUMEN}

Introducción: El sistema de Procesamiento Numérico y Cálculo (PNC) está compuesto por distintos elementos que funcionan de manera independiente. Objetivo: determinar las relaciones entre el razonamiento numérico y el cálculo. Pacientes y método: se evalúa el razonamiento numérico y el cálculo en dos pacientes con daño cerebral adquirido. Primero, una paciente con lesión frontal que presenta disfunción ejecutiva y segundo, un paciente con lesión perisilviana del hemisferio izquierdo y alteraciones en la producción del lenguaje. Resultados: el paciente con alteraciones lingüísticas es capaz de resolver problemas y razonar con números, sin embargo no puede realizar operaciones aritméticas simples si se le presentan de manera aislada, ni en forma oral ni escrita. Por otro lado, en el caso de daño frontal se observa el patrón inverso. Esta paciente realiza las tareas de cálculo de manera aislada o abstracta, tanto en forma oral como escrita, incluso realizando procedimientos de llevarse, pero no puede resolver problemas de razonamiento numérico simples. Discusión: el razonamiento numérico, es decir, la resolución de problemas mediante el uso de símbolos numéricos, es independiente de la realización de operaciones aritméticas de forma aislada o fuera de contexto, en abstracto. Estos resultados pueden ser explicados desde los modelos actuales.

Palabras clave: Afasia, cálculo, daño cerebral, disfunción ejecutiva, neuropsicología cognitiva, razonamiento numérico.

\section{ABSTRACT}

Introduction: Number processing and calculation system are composed by different elements that work independently. Objective: The objective is to determine relationship between number reasoning and calculation. The numerical reasoning and the calculation is evaluated in two patients with cerebral acquired damage. Patients and method: On one hand, a patient with frontal injury of left hemisphere that presents executive disfunction and on the other hand, a patient with injury of perisilvian area of the left hemisphere that presents alterations in the production of the oral language. Results: the patient with linguistic alterations is capable of solving problems and of reasoning with numbers, nevertheless he cannot execute simple arithmetical operations isolated (not in oral form not written). On the other hand, patient of frontal damage executes calculation isolated or abstract, both in oral and written form, even realizing procedures of taking, but she cannot solve problems of reasoning numerical simple. Discussion: number reasoning, that is, the resolution of problems by the use of numerical symbols, and isolated calculation work independently. These results can be explained from the actual processing number and calculation models.

Key words: Aphasia, Calculation, cerebral damage, executive dysfunction, cognitive neuropsychology, numerical reasoning. 


\section{Introducción}

Actualmente se considera que el procesamiento numérico y el cálculo (PNC) es un sistema formado por distintos elementos que pueden funcionar de manera independiente ya que son susceptibles de dañarse selectivamente (v.g., Alameda, Salguero y Lorca, 2007; Alsina y Sáiz, 2003, Brysbaert, 2005; Dehaene, 1992; McCloskey, Caramazza y Basili, 1985; Salguero, Lorca y Alameda, 2003, 2004).

Las últimas aportaciones desde la neuropsicología cognitiva confirman este supuesto y cada vez son más numerosas las evidencias a favor de la independencia entre los componentes que forman el sistema de PNC. Las operaciones aritméticas de sumar, restar y multiplicar están organizadas en el sistema cognitivo de manera autónoma (Harskamp y Cipolotti, 2001). El procesamiento de los signos aritméticos es susceptible de dañarse de manera selectiva, lo que implica su independencia funcional en el conjunto del sistema de PNC (Harskamp y Cipolotti, 2001; Salguero et al., 2003). La representación mental de la cantidad es independiente de otras habilidades numéricas como son el conocimiento numérico de carácter no cuantitativo o léxico (Alameda, Cuetos y Brysbaert, 2003; Salguero et al., 2004) así como el conocimiento numérico de tipo simbólico (Polk et al., 2001).

Los distintos modelos teóricos intentan explicar estos resultados con más o menos acierto. De ellos, el que se puede considerar con mayor poder explicativo es el denominado anatómico funcional propuesto por Dehaene y Cohen $(1995,1997)$. Este modelo postula la existencia de tres tipos de representaciones numéricas, con diferentes localizaciones cerebrales y encargadas de distintas tareas numéricas.

Forma visual-arábiga del número. Es la representación del número en forma arábiga y por tanto, es de carácter visual. Se localiza en las áreas occipito-temporales de ambos hemisferios. Se encarga de tareas numéricas como el procesamiento de dígitos arábigos, juicios de paridad y operaciones mentales de varios dígitos.

Estructura auditivo-verbal de la palabra del número. Es creada y manipulada por los módulos generales del lenguaje, y es la secuencia de palabras asociada al número. Se localiza en las áreas perisilvianas clásicas del lenguaje del hemisferio izquierdo y es responsable de tareas como nombrar y leer números en voz alta, contar, sumar y multiplicar datos simples.

Representación analógica de la magnitud. Las cantidades numéricas se representan como distribuciones de activación sobre una línea numérica analógica orientada de izquierda a derecha (o viceversa, según la cultura) que cumple la ley psicofísica de Weber. Se localiza en las áreas parietales inferiores de ambos hemisferios. Las tareas que realiza consisten en el procesamiento de cantidades analógicas, la comparación numérica (mayor-menor) y el cálculo aproximado o estimaciones numéricas. También está encargada del conocimiento numérico no cuantitativo (cualitativo o léxico).

En la Figura 1 se representa este modelo.

De acuerdo con este modelo existen dos vías para la resolución de una operación aritmética. Por una parte, los datos aritméticos exactos, principalmente los de las tablas de 


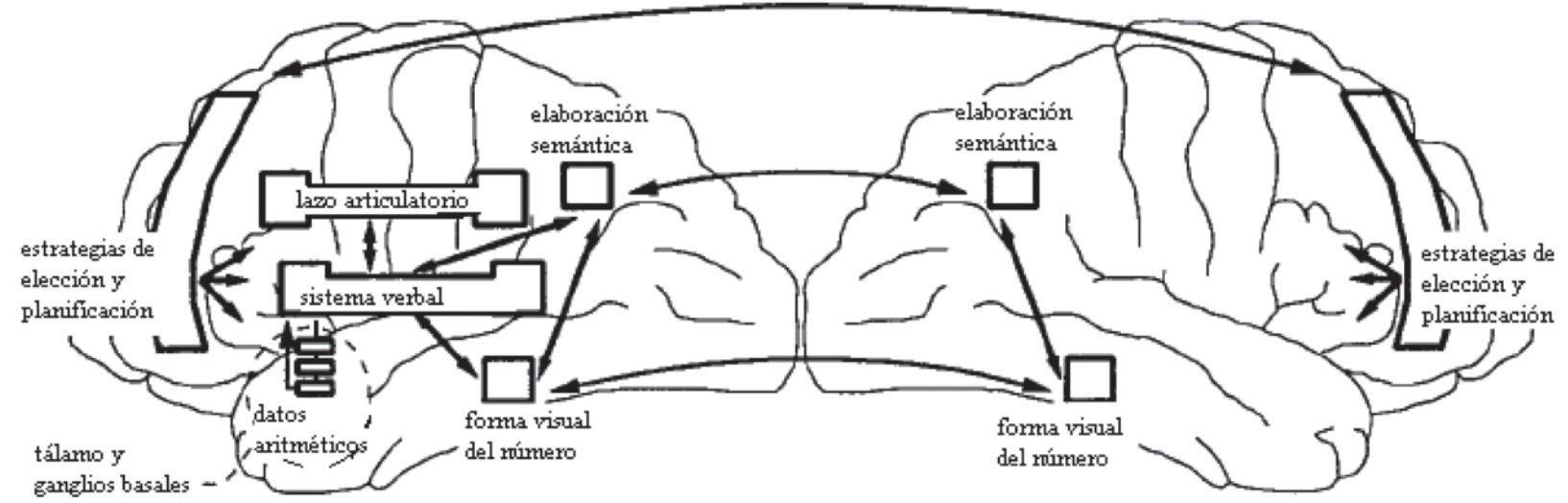

Figura 1. Representación del Modelo Anatómico Funcional.

sumar y multiplicar, que han sido aprendidos de forma memorística, por ejemplo, $2^{*} 2=4$, son recuperados en forma verbal, como automatismos verbales mientras que aquellos que no están almacenados de esta manera, se recuperarían mediante procesos de elaboración semántica, mediante la representación analógica de la magnitud, es decir, accediendo a la cantidad que representan los números y manipulándolos mentalmente.

Los objetivos de este trabajo son: primero, determinar las relaciones que existen entre la realización de operaciones de cálculo de manera aislada y su ejecución en el contexto de un problema de razonamiento numérico; $y$, segundo, determinar las bases neuroanatómicas diferenciales de cada manera de resolver una operación aritmética, ya sea contextualizada o en abstracto.

\section{Método Pacientes}

\section{A) Paciente con daño frontal}

Paciente ANB. Edad/Fecha de nacimiento: 38 años (14/5/1964). Mujer. Nivel educativo/ Años de escolaridad/Profesión: 17 años de
escolaridad/Licenciada en Filología Hispánica. Historia médica: Ingresa de urgencia con cuadro de cefalea frontotemporal de un mes de duración. Se diagnostica de quiste coloide del III ventrículo, con hidrocefalia reactiva. A los 3 días es intervenida quirúrgicamente. Se le realiza una craneotomía frontal izquierda, penetrando en el ventrículo lateral izquierdo a través de una corticotomía circular. La familia refiere desconexión total al principio del postoperatorio, de la que fue recuperándose poco a poco. Periodo de amnesia retrógrada a anterógrada (ésta última de mayor duración, aproximadamente un año). Refieren también problemas de memoria, repetición de ideas de forma obsesiva, y cambios de carácter. En informe psicológico se recoge presencia de déficit cognitivos relacionados con fallos de memoria e inadecuada adaptación a situaciones novedosas. Comienza una psicoterapia, pero abandona debido a la no evolución positiva. Derivada a Salud Mental, ante la persistencia de los fallos cognitivos y cambios en su carácter. Presenta síndrome extrapiramidal 3 años después. Durante todo este tiempo su estado 
de ánimo ha sido depresivo, con sentimientos de tristeza, desvaloración y desmotivación, acompañándose de sintomatología ansiosa. El servicio de Neurología, 4 años después, dictamina "secuelas cognitivas y conductuales tras intervención de quiste coloidal".

Evaluación neuropsicológica: Atención: focalizada y sostenida conservadas. Atención dividida alterada. Velocidad del procesamiento de la información: enlentecida significativamente. Funciones premotoras: programación, coordinación e integración del acto motor se encuentran conservadas. No comete errores en alternancias motoras y coordinación. Las funciones práxicas de tipo ideomotor e ideacional: preservadas. Orientación: temporal, del lugar y personal conservadas. Funciones visoperceptivas: capacidades visoespaciales y visomotoras, por debajo de la normalidad. Capacidades lingüísticas: expresión, articulación, repetición, y escritura normales. Comete más fallos en comprensión oral (sin llegar a ser incapacitante) que en escrita. Sin embargo su fluidez verbal y capacidad de denominación se encuentran disminuidas para su grupo de edad y educación equivalente. Memoria y aprendizaje: memoria de trabajo y manipulación mental de la información: se detectan dificultades. Importantes problemas para aprender material novedoso de naturaleza verbal. En términos generales, su curva de aprendizaje no sigue una distribución normal. Se detecta un índice nulo de recuerdo consistente, no llegando a recordar en ningún momento la totalidad de la información. No existe ganancia mnésica a lo largo de los ensayos. Comete numerosas intrusiones que dificultan el proceso de apren- dizaje. Los fallos hacen referencia al proceso de codificación del material, por lo que influye negativamente en el almacenamiento, así como en la recuperación de la poca información aprendida. No es capaz de almacenar información a largo plazo. En memoria visual, la paciente es capaz de retener a la media hora lo que aprendió de manera inmediata, por lo que apuntamos a fallos en su memoria visual inmediata más que a largo plazo. Los errores los comete a la hora de retener de manera inmediata los detalles aislados del diseño, desajustes característicos sobre todo del hemisferio cerebral izquierdo. Los resultados del Test de Retención Visual de Boston indican marcado deterioro cognitivo. Funciones ejecutivas: alteradas (Figura Compleja de Rey, recuerdo inmediato, Pc: 15; Matrices progresivas de Raven, $\mathrm{Pc}<1$ ). En la esfera psicosocial y emocional se producen cambios en personalidad debido a la enfermedad: alta frustración e irritabilidad, tristeza y desmotivación, ideas obsesivas, nerviosismo y marcada preocupación. Se descarta evidencia de depresión.

B) Paciente con daño en áreas perisilvianas del hemisferio izquierdo

Paciente PP. Edad/Fecha de nacimiento: 54 años (10/11/1950). Varón. Nivel educativo/Años de escolaridad/Profesión: 6 años de escolaridad/Empresario. Historia médica: Antecedentes de hipertensión arterial (HTA). Ingresa en el hospital por urgencias el 27 de marzo de 2000, con un cuadro de pérdida motora y sensitiva en miembros derechos (con previos episodios los días anteriores). Exploraciones: en EEG se visualizan ondas thetas-delta hipervoltadas en áreas parieto-temporales izquierdas. TAC craneal, muestra 
infarto isquémico en parietal izquierdo. RM craneal: infarto temporo-parieto-occipital izquierdo; pequeño infarto en protuberancia; focos isquémicos en corona radiada derecha y pedúnculo cerebral derecho; en ECO-doppler se detecta oclusión en origen de arteria carótida interna izquierda sin hallazgos patológicos en carótida derecha; en el EEG (48 horas después), concluye la afectación bioeléctrica cerebral lentificada a nivel de áreas parieto-temporales izquierdas. Es diagnosticado de trombosis de carótida interna izquierda en su origen, infarto cerebral en territorio de dicha arteria. Infartos lacunares cerebrales en otros territorios vasculares. En la arteriografía realizada en abril se confirma la oclusión del origen de la arteria carótida izquierda. En TAC de mayo de 2000 muestra hipodensidad a nivel parietal izquierdo. Recibe el alta en mayo de 2000, con diagnóstico de trombosis de arteria de carótida izquierda, con infarto cerebral en territorio de la citada arteria; hemiplejia derecha; HTA y afasia mixta. Evaluación neuropsicológica: Atención: déficit en atención sostenida y dividida. Reducción en la velocidad del procesamiento de la información.
Hemiagnosia homónima del campo visual derecho. Ligera alteración visoespacial y visoconstructiva. Lenguaje: moderada alteración de la fluidez verbal, denominación y repetición. Alexia. Agrafia. Acalculia. Memoria: alteración de la memoria verbal y visual. Apraxia ideomotora e ideacional. Hemiplejia derecha. Funciones ejecutivas: ligera alteración, ligera desinhibición social, impulsividad, rigidez mental y baja tolerancia a la frustración.

\section{Procedimiento}

A ambos pacientes se les aplican las mismas tareas para la evaluación del razonamiento numérico y el cálculo aislado. La aplicación es individual. Estas pruebas forman parte de la batería de Evaluación de procesamiento numérico y cálculo (Salguero y Alameda, 2007) y los datos relativos a la frecuencia se tomaron de Cuetos y Alameda (1997). En la Tabla 1 se presentan los resultados del grupo control en estas pruebas. Para el análisis estadístico de los datos se ha realizado una diferencia de propor-

Tabla 1

Pruebas de evaluación del cálculo.

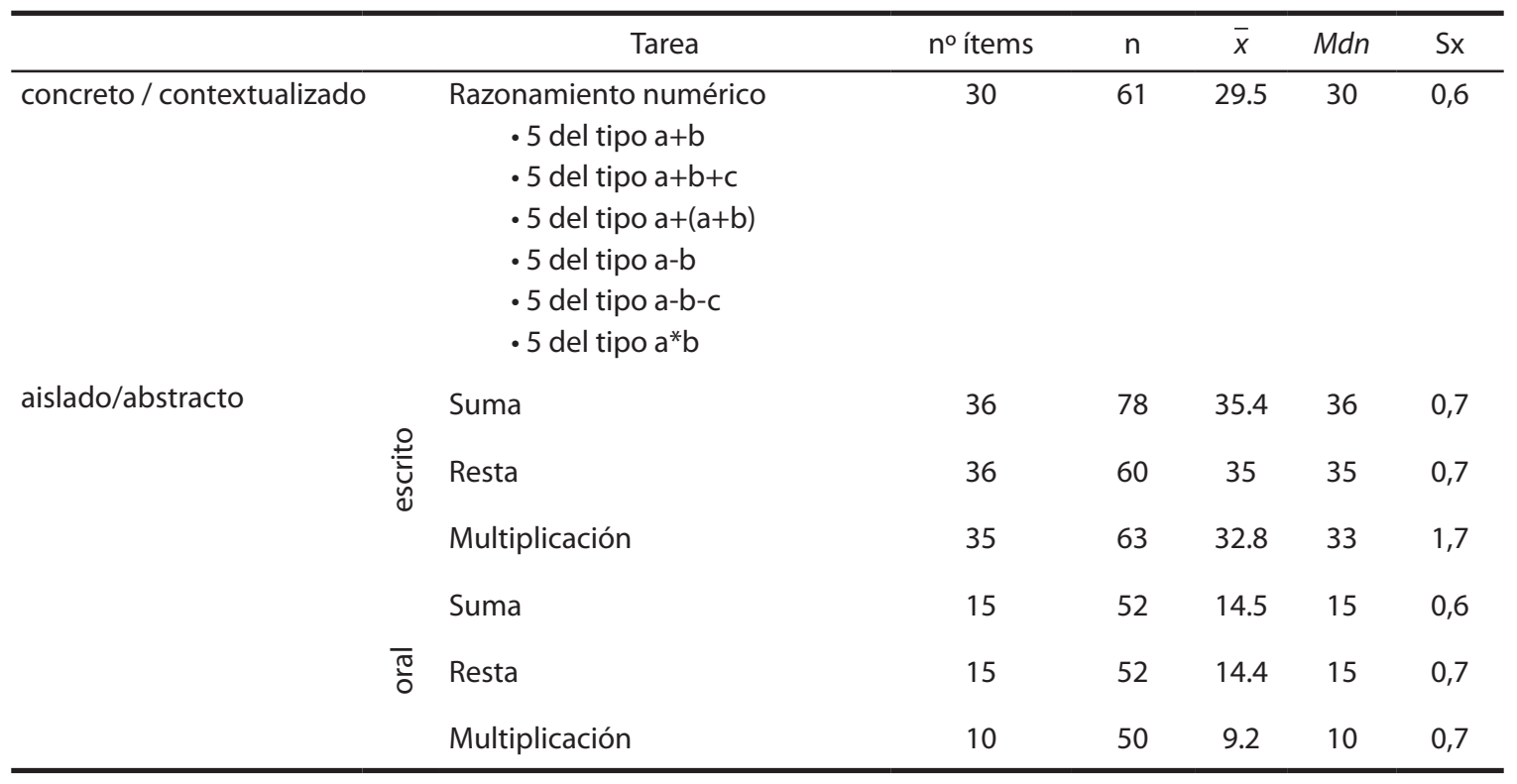


ciones, procedimiento con el que se compara la ejecución de cada uno de los pacientes con el grupo control (Moore y McCabe, 2001; Pryce, 2005).

\section{Resultados}

En la Tabla 2 se presentan los resultados de estos pacientes en cada prueba.

La paciente ANB no presenta alteraciones en las tareas de cálculo aislado, los resultados son iguales a los del grupo control en las distintas operaciones aritméticas evaluadas, suma, resta y multiplicación, tanto en la modalidad escrita como en la oral. Sin embargo, es incapaz de realizar alguno de los tipos de problemas de razonamiento numérico. Las diferencias con el grupo control son significativas en los proble- mas de tipo $a-b-c, a+(a+b)$ y a*b, así como en el total de la prueba $(z=4,08 ; p<0,01)$. Es decir, ANB no es capaz resolver un problema de razonamiento numérico sencillo, por ejemplo, "un libro vale 5 euros, ¿cuánto valen 4 libros?", pero en tarea de cálculo oral, no presenta dificultad para responder ¿cuánto es $5^{*} 4$ ?, y en tareas de cálculo escrito, realiza correctamente este tipo de multiplicaciones, e incluso otras más complejas, por ejemplo, $73 * 5$, en la que hay que multiplicar 2 dígitos por un dígito y ejecutar el procedimiento de llevarse.

Por otro lado, el paciente PP con alteraciones en la producción del lenguaje como consecuencia de lesiones perisilvianas izquierdas presentan el patrón de ejecución inverso al que hemos visto para los lesionados frontales. En

Tabla 2.

Resultados de los pacientes en cada prueba.

\begin{tabular}{|c|c|c|c|}
\hline PRUEBAS & & ANB & PP \\
\hline \multirow{3}{*}{ RAZONAMIENTO NUMÉRICO } & $\% \mathrm{PD}$ & 50 & 100 \\
\hline & z & 4,08 & $-0,99$ \\
\hline & $p$ & $<0,01$ & 0,32 \\
\hline \multirow{3}{*}{ SUMA ESCRITA } & $\% \mathrm{PD}$ & 97 & 69 \\
\hline & z & 0,00 & 3,16 \\
\hline & $p$ & 1,00 & $<0,01$ \\
\hline \multirow{3}{*}{ RESTA ESCRITA } & $\% \mathrm{PD}$ & 100 & 96 \\
\hline & z & $-1,31$ & $-0,43$ \\
\hline & $p$ & 0,18 & 0,66 \\
\hline \multirow{3}{*}{ MULTIPLICACIÓN ESCRITA } & $\% \mathrm{PD}$ & 100 & 66 \\
\hline & z & $-1,16$ & 2,18 \\
\hline & $p$ & 0,24 & 0,02 \\
\hline \multirow{3}{*}{ SUMA ORAL } & $\% \mathrm{PD}$ & 86,5 & 60 \\
\hline & z & 0,6 & 2,15 \\
\hline & $p$ & 0,54 & 0,03 \\
\hline \multirow{3}{*}{ RESTA ORAL } & $\% \mathrm{PD}$ & 80 & 46,5 \\
\hline & z & 1,07 & 2,78 \\
\hline & $p$ & 0,28 & $<0,01$ \\
\hline \multirow{3}{*}{ MULTIPLICACIÓN ORAL } & $\% \mathrm{PD}$ & 80 & 3 \\
\hline & z & 0,62 & 2,73 \\
\hline & $p$ & 0,53 & $<0,01$ \\
\hline
\end{tabular}


la tarea de Razonamiento Numérico los resultados son de un $100 \%$ aciertos. Sin embargo, presenta importantes dificultades en las tareas de cálculo, tanto en forma oral como escrita, excepto en la resta escrita. En suma y multiplicación escritas las diferencias con el grupo control son significativas $(z=3,16 ; p=0,001$ y $z$ $=2,18 ; p=0,028$, respectivamente). En cálculo oral PP presenta alteraciones tanto en suma ( $z$ $=2,15 ; p=0,03)$, resta $(z=2,78 ; p<0,01)$ como multiplicación $(z=2,73 ; p=0,01)$.

Estos resultados de PP por un lado, y de ANB por otro, podrían indicar la existencia de una doble disociación entre calcular de forma aislada y calcular en el contexto de un problema de razonamiento numérico, es decir, una doble disociación entre el cálculo contextualizado y el cálculo aislado, y por tanto, su independencia funcional y/o estructural. En la Figura 2 se pueden observar las diferencias respecto al grupo control entre ambos conjuntos de pacientes. de un procesamiento de información que es complejo y en el que se incluye la decisión de la operación que es necesaria para resolver el problema. De acuerdo con Miranda y GilLlario (2001) son varias las fases del proceso de resolución de un problema, entre las que se incluye la elección de una estrategia y de la operación aritmética que permita llegar al resultado partiendo de los datos disponibles. Para desarrollar correctamente estas etapas son necesarios los mecanismos de planificación cognitiva. En otras palabras, el cálculo en un problema de razonamiento sencillo (por ejemplo, del tipo $\mathrm{a}^{*} \mathrm{~b}$ ) es el último paso del proceso. ANB es capaz de realizar el cálculo, puesto que si se le pregunta fuera de contexto cuál es el resultado de $a * b$ responde correctamente, pero cuando se le plantea en el contexto de una situación cotidiana, en un problema, no es capaz de resolverlo porque no puede llegar a esta última fase, las dificultades son previas, se localizan en los proce-

\section{Discusión y conclusiones}

La paciente ANB realiza correctamente las operaciones aritméticas de sumar, restar y multiplicar tanto en forma oral como escrita, pero no es capaz resolver un problema de razonamiento numérico sencillo. Una posible explicación a este resultado es que el razonamiento numérico implica en primer lugar "pensar" con números y luego, por último, calcular, es decir, operar con esos números, pero requiere previamente

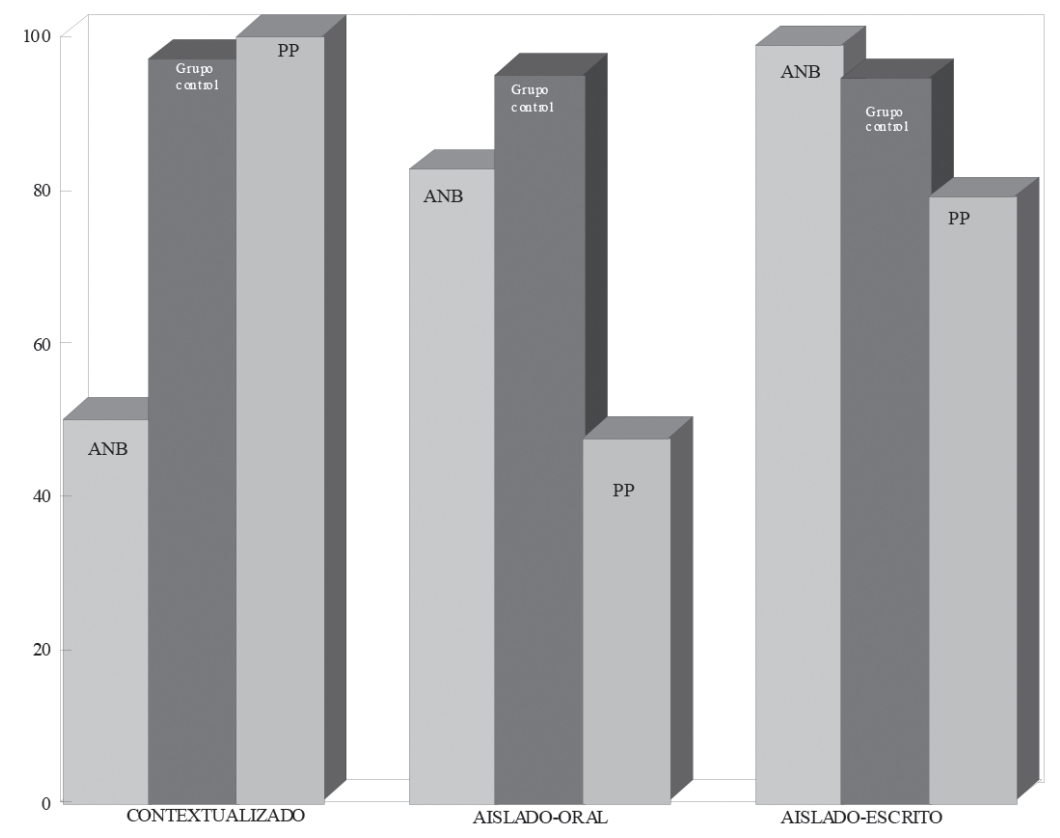

Figura 2.- Resultados en cálculo aislado (oral y escrito) y en cálculo contextualizado (porcentajes de aciertos). 
sos de planificación y se deben posiblemente a la disfunción ejecutiva que presenta, como consecuencia del daño frontal. Estos resultados no pueden explicarse por déficit en la comprensión lingüística, ya que la paciente comprende sin dificultad el lenguaje oral en general, y en concreto comprende perfectamente los enunciados de los problemas que se le presentan oralmente, pero afirma que a pesar de comprender lo que se le está preguntando no puede responder ("no puedo, no sé, sé lo quiere decir pero no sé, no puedo, no puedo...."). Por tanto, la alteración tiene lugar en el proceso de "pensar con números", de planificar y seleccionar la estrategia que debe seguir para resolver el problema. Este resultado confirma que el cálculo es sólo un paso del proceso de razonamiento numérico. Sin embargo, esta dificultad de planificación de la paciente no se puede considerar generalizada ya que conserva las funciones ejecutivas necesarias para desenvolverse en la vida cotidiana, es capaz de realizar de forma autónoma actividades como vestirse, así como decidir qué es lo que le apetece hacer en un momento determinado.

Por otro lado, en el caso de PP con lesión en las áreas perisilvianas izquierdas, y grave afectación en la producción del lenguaje, se observa el patrón inverso, es decir, tiene dificultades graves para la resolución de operaciones aritméticas de manera aislada pero es capaces de resolver cálculos que se le presentan en el contexto de un problema de razonamiento, es como si se produjera un efecto de facilitación del contexto.

El modelo de Dehaene y Cohen (1995, 1997), como se ha comentado, plantea que una operación aritmética simple se puede resolver a través de dos rutas: la lingüísticamemorística, o mediante elaboración semántica. Cuando se presentan los operandos en el contexto de un problema podría ser que se facilitara la activación de la vía semántica. Por esta razón los pacientes que tiene alterada la vía lingüística presentan mejores ejecuciones en razonamiento numérico que en cálculo aislado. En las operaciones contextualizadas se produciría una activación de los procesos de elaboración semántica, algo que no sucedería en cálculo aislado. Esto nos permiten afirmar que las funciones numéricas no son simétricas en ambos hemisferios al igual que sucede con el lenguaje.

Por otra parte, el efecto inhibidor encontrado en la paciente $A N B$, se explicaría también desde el modelo anatómico funcional (Dehaene y Cohen, 1997) como consecuencia de las alteraciones en las funciones ejecutivas, debido a las lesiones en las áreas frontales. Es lo que estos autores denominan selección de estrategia y planificación.

Como conclusión se podría afirmar que los procesos cognitivos implicados en el cálculo aislado y en el razonamiento numérico son funcionalmente independientes.

\section{Bibliografía}

Alameda, J. R., Cuetos, F., \& Brysbaert, M. (2003). The number 747 is named faster after seeing Boeing than after seeing Levi's: Associative priming in the processing of multidigit Arabic numerals. The Quarterly Journal of Experimental Psychology, 56A, 1009-1019.

Alameda, J. R., Salguero, M. P. \& Lorca, J. A. (2007). Conocimiento numérico cuanti- 
tativo y léxico: evidencia de doble disociación. Psicothema, 19, 381-387.

Brysbaert, M. (2005). Number recognition in different formats. En J. I. D. Campbell (Ed.), Handbook of Mathematical Cognition (pp. 23-42). Nueva York: Psychology Press.

Cuetos, F. y Alameda, J. R. (1997). El efecto frecuencia en el procesamiento de los números. Un recuento de frecuencias. Cognitiva 9, 207-223.

Dehaene, S. (1992). Varieties of numerical abilities. Cognition, 44, 1-42.

Dehaene, S. \& Cohen, L. (1995). Towards an anatomical and functional model of number processing. Mathemathical Cognition, 1, 83120.

Dehaene, S., \& Cohen, L. (1997). Cerebral pathways for calculation: double dissociation between rote verbal and quantitative knowledge of arithmetic. Cortex, 33, 219-250.

Ferro, J. M., \& Botelho, M. A. S. (1980). Alexia for arithmetical sign. A cause of disturbed calculation. Cortex, 16, 175-80.

Harskamp, N. J., \& Cipolotti, L. (2001). Selective impairments for addition, subtraction and multiplication. Implications for the organisation of arithmetical facts. Cortex, 37, 363-388.

McCloskey, M., Caramazza, A., \& Basili, A. (1985). Cognitive mechanisms in number processing and calculation: Evidence from dyscalculia. Brain and Cognition, 4, 171-96.

Miranda, A. \& Gil-Llario, M. D. (2001). Las dificultades de aprendizaje en las matemáticas: concepto, manifestaciones y procedimientos de manejo. Revista de Neurología Clínica, 2, 55-71.

Moore, D. S., \& McCabe, G. P. (2001). Introduction to the Practice of Statistics. Nueva York: WH Freeman.

Polk, T. A., Reed, C. L., Keenan, J. M., Hogarth, P., \& Anderson, C. A. (2001). A dissociation between Symbolic Number Knowledge and Analogue Magnitude Information. Brain and Cognition, 47, 545-563.
Pryce, G. (2005). Inference and Statistics in SPSS. Glasgow: GeeBeeJey Publishing.

Salguero, M. P. y Alameda, J. R. (2007). Batería para la Evaluación del procesamiento numérico y el cálculo. Huelva: Hooverand S.L.

Salguero, M. P., Lorca, J. A. \& Alameda, J. R. (2003). Procesamiento numérico y cálculo: evidencia de un caso desde la Neuropsicología Cognitiva. Revista de Neurología, $36,817-820$.

Salguero, M. P., Lorca, J. A. \& Alameda, J. R. (2004). Independencia funcional del conocimiento numérico léxico y la representación de la magnitud: Evidencia de un caso. Revista de Neurología, 39, 1038-1042. 
